\title{
Percolation of Monte Carlo Clusters
}

\author{
W.G. Wanzeller ${ }^{1}$, A. Cucchieri ${ }^{2}$, T. Mendes ${ }^{2}$, and G. Krein ${ }^{1}$ \\ ${ }^{1}$ Instituto de Física Teórica, Universidade Estadual Paulista, Rua Pamplona 145, 01405-900, São Paulo, SP, Brasil \\ ${ }^{2}$ Instituto de Física de São Carlos, Universidade de São Paulo, P.O. Box 369, 13560-970, São Carlos, SP, Brasil
}

Received on 15 August, 2003.

\begin{abstract}
Percolation theory is of interest in problems of phase transitions in condensed matter physics, and in biology and chemistry. More recently, concepts of percolation theory have been invoked in studies of color deconfinement at high temperatures in Quantum Chromodynamics. In the present paper we briefly review the basic concept of percolation theory, exemplify its application to the Ising model, and present the arguments for a possible relevance of percolation theory to the problem of color deconfinement.
\end{abstract}

\section{Percolation}

In order to explain the concept of percolation, let us consider Fig. 1. The figure represents pictorially an electrical circuit composed of a set of conducting spheres, represented by $(\bullet)$ and a set of insulating spheres, represented by (o). An electrical current will flow from the top plate to the bottom plate and the bulb $(\otimes)$ will light once enough conducting spheres are present in the circuit. That is, there will be a "percolating" electrical current through the system when a conducting sphere from the top is continuously connected to a conducting sphere on the bottom. It is intuitively clear that percolation will happen once a minimum of conducting spheres is present in the system, i.e. there is a critical value for the concentration of conducting spheres for which a "percolating cluster" will be formed.

(a)

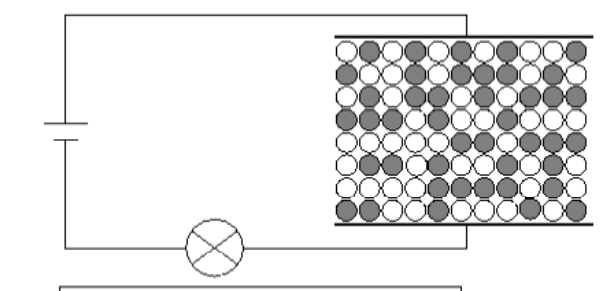

(b)

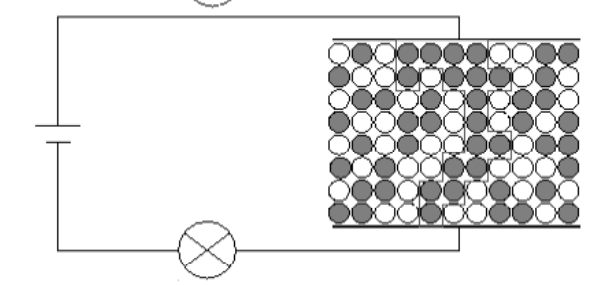

Figure 1. Pictorial representation of percolation. When a continuous chain from the top to the bottom is formed as in (b), there will be a percolating current and the light bulb $(\otimes)$ will turn on.

The existence of a critical value for percolation and the consequent formation of a percolation cluster can be illustrated as follows. Let us consider a square lattice of volume $L \times L$. For each lattice site, we attribute a probability $p$ that the site will be occupied, and a probability $1-p$ that it is empty. A "cluster" is defined as groups of neighboring occupied sites. Suppose now that one fixes a value of $p$ and at each site of the lattice we draw a random number $r$. If $r \leq p$, then one considers that the site is occupied, if $r>p$, the site is considered empty. Groups of neighboring occupied sites are collected to form a cluster. At the end, one checks if there is at least one cluster that has percolated, that is, if an occupied site from the top of the lattice belonging to some cluster is continuously connected to an occupied site at the bottom of the lattice. Then, the process is repeated many times. Each complete visit to all sites of the lattice is called a "sweep". Next, we define a percolation probability as

$$
\langle P\rangle=\frac{1}{N} \sum_{j=1}^{N} C_{j}
$$

where $C_{j} \equiv 1(0)$ if a percolating cluster has (has not) occurred during the sweep $j$, and $N$ is the number of sweeps.

In Fig. 2 we plot $\langle P\rangle$ for different values of $p$ for $L=$ $100,250,400,600$ and 1000 , using free boundary conditions. One sees that there exists a transition region around a certain value of $p$ where the percolation probability changes from a small value to a value that becomes close to one for large lattices. It is clear that as the lattice becomes larger and larger, the transition region becomes sharper and sharper, with a well defined transition point $p_{c}$. In the limit of an infinite lattice, for the present case of a two-dimensional cubic lattice the value of $p_{c}$ is found to be [1]

$$
p_{c}=0.592746
$$

Clearly the numerical simulations, for large lattices, give $p_{c}$ very close to this value. To be more precise, using the principle of finite-size-scaling, our simulations give [2]

$$
p_{c}=0.5927 \pm 0.0001
$$




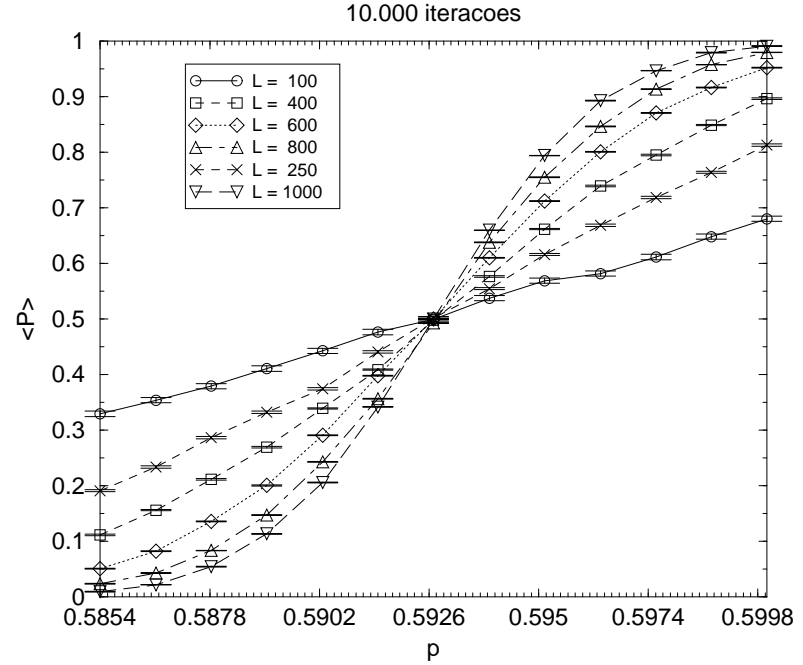

Figure 2. $\langle P\rangle$ for different lattices sizes.

\section{Percolation theory in the Ising Model}

The magnetic phase transition that occurs in the Ising model (for dimensions larger than 1) can be related to a percolation phenomenon. The relation was established in two major steps. The first step was given in 1972 by Fortuin and Kasteleyn [3], who showed that it is possible to rewrite the partition function of the Ising model as a sum over configurations of spin clusters, instead of the usual sum over spin configurations, as follows

$$
\mathcal{Z}=\sum_{\{S= \pm 1\}} \sum_{\{n\}}\left\{\prod_{<i, j>}^{n_{i j}=1} p_{i j} \delta_{S_{i} S_{j}}\right\}\left\{\prod_{<i, j>}^{n_{i j}=0}\left(1-p_{i j}\right)\right\}
$$

where

$$
p_{i j}=1-\exp (-2 J \beta),
$$

is the probability that a bond between spins at neighboring sites $i$ and $j$ occurs, and $n_{i j}=1(0)$ indicates that there is (there is not) a bond between spins at $i$ and $j$. Note that a bond may be placed only between spins of the same value. In Eq. (5) $\beta=1 / k T$, where $k$ is the Boltzmann constant and $J$ is the spin-spin strength, defined in the Ising Hamiltonian as

$$
H=-J \sum_{\langle i j\rangle} S_{i} S_{j}
$$

where $\langle i j\rangle$ means nearest neighbors and $S_{i}= \pm 1 / 2$ are spins at site $i$. A spin cluster can therefore be naturally defined as the collection of spins that are continuously bound. Note that now the probability of a spin to belong to a cluster depends on the temperature (and the strength $J$ ) as in Eq. (5). The next step was given in 1980 by Coniglio and Klein [4] when they showed, using renormalization group techniques that the magnetic critical temperature $T_{c}$ of the
Ising model determines the critical value for the occurrence of a percolating cluster, with $p_{c}$ given by the formula $p_{c}=1-\exp \left(-2 J / k T_{c}\right)$. The Fortuin-Kasteleyn clusters were later used (in 1987) by Swendsen and Wang [5] as a very efficient way of performing global spin updates in Monte Carlo simulations of spin models.

In Fig. 3 we show results of our Monte Carlo simulation for the percolation probability $\langle P\rangle$, defined in Eq. (1). In the figure we show results for lattice sizes $L=100,400,600$ and 800. A detailed description of the Swendsen-Wang algorithm used, the reasons for using it, comparisons with other Monte Carlo algorithms, and the error analysis performed can be found in Ref. [2]. The exact critical temperature of the two-dimensional Ising model is (for $J=1$ ) $T_{c}=2.269185$. Clearly, our simulations show that the percolation temperature, determined with the use of Eq. (5) shows a good agreement with the exact value. Specifically, we obtained

$$
T_{c}=2.2691 \pm 0.0001 \text {. }
$$

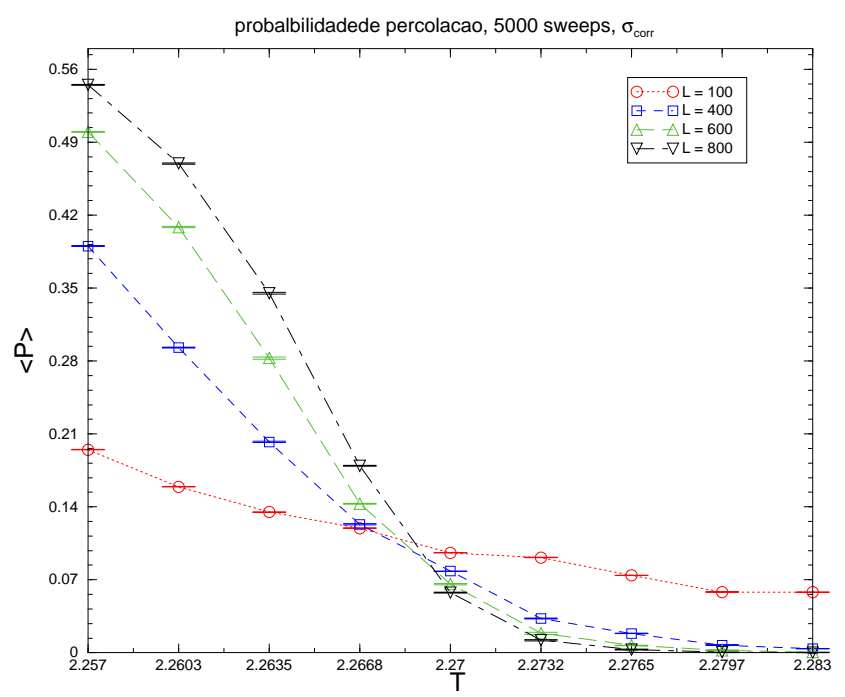

Figure 3. $\langle P\rangle$ for $L=100,400,600$, and 800 using the SwensenWang cluster algorithm.

\section{Percolation Theory and Deconfine- ment in QCD}

As said above, percolation theory is of interest in several areas of natural science. Our motivation in this theory is driven basically by its possible relevance to the problem of color deconfinement in Quantum Chromodynamics (QCD). The connection between the two phenomena can be argued as follows $[6,8]$.

Let us consider the formulation of a pure $S U(N)$ gauge theory on an Euclidean lattice with $N_{\sigma}$ points in each spatial direction and $N_{\tau}$ points in the time direction. The partition function at finite temperature can be written as

$$
\mathcal{Z}\left(N_{\sigma}, N_{\tau} ; g^{2}\right)=\int \prod_{\text {links }}[d U] \exp \{-S[U]\}
$$


where the integration is over link variables $U_{\mu}$,

$$
U_{\mu}=\exp \left[-i g a A_{\mu}(\vec{x}, t)\right]
$$

with $a$ the lattice spacing and $\mu$ the space-time direction, and $S[U]$ is the Wilson action given by

$$
S[U]=\frac{2 N}{g^{2}} \sum_{P}\left[1-\frac{1}{N} \operatorname{Re} \operatorname{Tr}\left(U_{P}\right)\right] .
$$

Here $\sum_{P}$ means that the sum is done over all plaquettes $U_{P}$, where a plaquette is the basic circuit of four U's [7].

At finite temperatures, the gauge field configurations are periodic in the "time" interval $[0, \beta]$. This periodicity leads to a global symmetry that is important for the phase structure of the theory at finite temperature. Because of such a symmetry, gauge transformations in the time direction only need to be periodic up to an element $z$ of the center $Z(N)$ of $S U(N)$. The action remains invariant if the gauge transformation $G$ in $S U(N)$ satisfies the condition

$$
G(\vec{x}, 0)=z G(\vec{x}, \beta),
$$

for all spatial points $x$. Now, since an element of $Z(N)$ is given by

$$
z_{n}=e^{\frac{2 \pi i n}{N}}, \quad n=0,1, \cdots N-1,
$$

it is easy to show that the quantity, known as the Polyakov loop,

$$
L_{x}=\frac{1}{N} \operatorname{Tr} \prod_{t=1}^{N_{\tau}} U_{4}(\vec{x}, t)
$$

changes as

$$
L_{x} \rightarrow z L_{x}
$$

under a gauge transformation as in Eq. (11). An average over the lattice and field configurations, $\bar{L}=\left\langle L_{x}\right\rangle$, which indicates the state of the system, does not change this result. One can show that the free energy $F$ of a very heavy quark immersed in the QCD vacuum is related to $\bar{L}$ as

$$
\bar{L} \sim e^{-\beta F} .
$$

In the confined phase, one expects $F=\infty$, and therefore $\bar{L}=0$, and the transformation given in Eq. (14) leaves $\bar{L}$ invariant. The transition from a confined to a deconfined pure gauge $S U(N)$ field theory can therefore be characterized by the breaking of the global center $Z(N)$ symmetry, since in the deconfined phase $\bar{L} \neq 0$ breaks the invariance of the theory. Studies of lattice gauge theories show that such a phase transition indeed takes place.

Now, it is natural to speculate whether the deconfinement phase transition of $S U(N)$ gauge theories can be studied in analogy to the phase transitions in the much simpler $Z(N)$ spin systems. In particular, it has been confirmed by lattice gauge simulations that the critical exponents of $S U(2)$ gauge theory and $Z(2)$ spin system fall in the same universality class. From the discussion in Section II, it is very natural to conjecture that color deconfinement could be a percolation phenomenon.

Recently, Satz and Fortunato [6, 8] have undertaken percolation studies for $S U(2)$ gauge theories. This was made possible by the use of the Green-Karsch [9] effective theory which allows to write the partition function of the theory, in the strong coupling limit and neglecting spacelike plaquettes, as

$$
\mathcal{Z} \sim \int \Pi_{x} d L_{x} \sqrt{1-L_{x}^{2}} \exp \left(\beta^{\prime} \sum_{i j} L_{i} L_{j}\right)
$$

where $L_{i}$ is the value of the Polyakov loop at site $i$, the sum is over nearest neighbors, and

$$
\beta^{\prime}=4\left(\frac{\beta}{4}\right)^{N_{\tau}}
$$

From this, one can define a bond probably (between sites with $L_{i}, L_{j}$ of the same sign) as

$$
p_{i j}=1-\exp \left\{-2 \beta^{\prime} L_{i} L_{j}\right\} .
$$

\section{Conclusions and Future Perspec- tives}

Our aim in this communication was to present the arguments in favor of a percolation description of the deconfinement transition in QCD. We started with a brief review the basic concept of percolation theory through a simple example of site percolation. Then, we proceeded by showing as the magnetic phase transition that occurs in the twodimensional Ising model can be understood as a percolation phenomenon. In the last section, we presented the connection between $S U(2)$ gauge theory and effective spin system, as argued by Satz and Fortunato [6, 8].

Using percolation theory, we have measured the critical temperature and the critical exponent for the percolation process and have compared with the corresponding critical magnetic observables, in conformity with Coniglio and Klein previsions [4]. The conclusion is that the percolation process and the magnetic phase transition of the $2 \mathrm{~d}$-Ising model are in the same universality class. Some of the results shown here are not new, but all numerical codes employed in the simulations and error estimates are original.

We are currently extending our percolation studies to the continuous $O(4)$ spin model with magnetic field. Another direction we are pursuing is the evaluation of dinamic critical exponents [10].

\section{Acknowledgments}

This work was partially supported by CAPES, CNPq and FAPESP (Brazilian financial agencies). 


\section{References}

[1] D. Stauffer and A. Aharony, Introduction to Percolation Theory (Taylor \& Francis, 1992).

[2] W.G. Wanzeller, Estudo de Percolação de Clusters de Monte Carlo para o Modelo de Ising Bi-dimen-sional, Master-Dissertation, Instituto de Física Teórica, IFT/UNESP D.002/03 (2003) (unpublished).

[3] C. M Fortuin and P.W. Kasteleyn, Physica 57, 536 (1972).

[4] A. Coniglio and W. Klein, J. Phys. A 13, 2775 (1980).
[5] R. Swendsen and J-S. Wang, Phys. Rev. Lett. 58, 1 (1987).

[6] H. Satz, Comp. Phys. Commun. 147, 46 (2002); Fortsch. Phys. 50, 541 (2002); Nucl. Phys. A 681, 3 (2001).

[7] H.J. Rothe, Lattice Gauge Theories: An Introduction (World Scientific, 1998).

[8] S. Fortunato, Percolation and Deconfinement in SU(2) Gauge Theory. PhD Thesis, Physics Faculty, University of Bielefeld.

[9] F. Green and F. Karsch, Nucl. Phys. B 238, 297 (1984).

[10] M. Suzuki, Prog. Theor. Phys. 58, 1142 (1977). 